\title{
An Antiviral Mechanism Investigated with Ribavirin as an RNA Virus Mutagen for Foot-and-mouth Disease Virus
}

\author{
Chao-jiang Gu, Cong-yi Zheng*, Qian Zhang, Li-li Shi, Yong Li and San-fu Qu \\ State Key Laboratory of Virology, College of Life Sciences, Wuhan University, Wuhan 430072, China
}

Received 29 June 2005, Accepted 17 August 2005

To prove whether error catastrophe /lethal mutagenesis is the primary antiviral mechanism of action of ribavirin against foot-and-mouth disease virus (FMDV). Ribavirin passage experiments were performed and supernatants of $R p_{1}$ to $R p_{5}$ were harvested. Morphological alterations as well as the levels of viral RNAs, proteins, and infectious particles in the BHK-21 cells infected using the supernatants of $R p_{1}$ to $R p_{5}$ and control were measured by microscope, real-time RT-PCR, western-blotting and plaque assays, respectively. The mutation frequency was measured by sequencing the complete $\mathrm{P1}$ - and $3 \mathrm{D}$-encoding region of FMDV after a single round of virus infection from ribavirintreated or untreated FMDV-infected cells. Ribavirin treatment for FMDV caused dramatically inhibition of multiplication in cell cultures. The levels of viral RNAs, proteins, and infectious particles in the BHK-21 cells infected were more greatly reduced along with the passage from $R p_{1}$ to $R p_{5}$, moreover, nucleocapsid protein could not be detected and no recovery of infectious virus in the supernatant or detection of intracellular viral RNA was observed at the $\mathrm{Rp}_{5}$-infected cells. A high mutation rate, giving rise to an 8-and11-fold increase in mutagenesis and resulting in some amino acid substitutions, was found in viral RNA synthesized at a single round of virus infection in the presence of ribavirin of $1000 \mu \mathrm{M}$ and caused a 99.7\% loss in viral infectivity in contrast with parallel untreated control virus. These results suggest that the antiviral molecular mechanism of ribavirin is based on the lethal mutagenesis/error catastrophe, that is, the ribavirin is not merely an antiviral reagent but also an effective mutagen.

Project supported by the Special Funds for Major State Basic Research of China (973), (NO. G1999011904)

\footnotetext{
*To whom correspondence should be addressed.

Tel: 86-27-68754001; Fax: 86-27-68753833

E-mail: CCTCC202@whu.edu.cn
}

Keywords: Antiviral mechanism, Error catastrophe, Footand-mouth disease virus, Lethal mutagenesis, Ribavirin, Viral extinction

\section{Introduction}

Ribavirin (1- $\beta$-D-ribofuranosy-1,2,4-triazole-3-carboxamide) discovered over 30 years ago is the first synthetic, broadspectrum antiviral nucleoside (Sidwell et al., 1972) which exerts antiviral activity against a wide range of DNA and RNA viruses (Snell, 2001). So far, it is currently used clinically as monotherapy to treat respiratory syncytial virus infection (Wyde, 1998; Krilov, 2001; Xu et al., 2004) as well as lassa fever virus infection (Andrei and De Clercq, 1993; Shane and Raul, 2002; De Clercq, 2004) and in combination with interferon- $\alpha$ to treat hepatitis $\mathrm{C}$ virus (HCV) infection (Cummings et al., 2001, Di Bisceglie et al., 2001, Maag et al., 2001). Ribavirin is phosphorylated by cellular adenosine kinase into ribavirin mono-, di-, and triphosphate (RMP, RDP and RTP, respectively) and exhibits its antiviral effect via direct and indirect mechanisms: First, RMP inhibits inosine monophosphate dehydrogenase (IMPDH), causing a decline of GTP concentration in the intracellular (Streeter, 1973) which potentially affects viral protein synthesis and limits replication of genome. Second, RTP inhibits directly some viral RNA-dependent RNA polymerase activity (Eriksson et al., 1977; Wray et al., 1985; Wray et al., 1985; Toltzis et al., 1988; Fernandez-Larsson et al., 1989). Meanwhile, RTP inhibits capping of viral mRNA through suppressing the 2'-Omethyltransferase activity (Delphine Benarroch et al., 2004), and as a substrate for a viral RNA capping enzyme (Isabelle and Martin, 2004). In addition, some mechanisms of action have been put forward, including RMP inhibition of guanylyltransferase activity (Isabelle and Martin, 2004) and inhibition of viral transcription (Eriksson et al., 1977; Cassidy and Patterson, 1989). It is known that RNA viruses encode the RNA polymerases which are used to replicate their genome. 
Owing to the inherently high error-rates and a lack of proofreading mechanisms of RNA polymerase, RNA viruses exist as a quasispecies- that is, an extremely heterogeneous population in which each member differs from another by a finite number of changes in the viral genome (Domingo et al., 1985). The error catastrophe theory as a corollary of quasispecies theory was put forward which predicts that, once the threshold of 'error catastrophe' is reached, a modest increase in mutation can kill a RNA virus population by causing a 'genetic meltdown' (Stuart, 1996). Recently, it was proved that ribavirin triphosphate was incorporated into viral RNA by the poliovirus RNA polymerase $3 \mathrm{D}^{\mathrm{pol}}$, enhancing mutation frequency by allowing base mismatches, which lead to lethal mutagenesis of viral genome (Shane et al., 2000), accounting for the observed antiviral effect. In this report, we aimed to investigate the antiviral mechanism by using ribavirin as the antiviral compound and FMDV as a model RNA virus by ribavirin passage experiments.

\section{Materials and Methods}

Reagents. Ribavirin purchased from Sigma was dissolved in DMEM to yield stock solution of $10 \mathrm{mmol} / \mathrm{L}$. The solution was sterilized by filtration $(0.22 \mu \mathrm{m}$ membrane filter). Stock solution was aliquoted, stored at $-20^{\circ} \mathrm{C}$ and diluted as needed.

Cells, viruses, and treatments. The origins of FMDVs of serotype $\mathrm{O}$ used for experiment was derived from Lanzhou Veterinary Research Institute, Chinese Academy of Agriculture sciences, lanzhou. The baby hamster kidney 21 cells (BHK-21) were provided by China center for type culture collection (CCTCC). BHK-21 cells were cultured in Dulbecco's modified Eagle's medium (DMEM) supplemented with 10\% heat-inactivated fetal bovine serum (FBS) and $1 \%$ penicillin-streptomycin at $37^{\circ} \mathrm{C}$ with $5 \% \mathrm{CO}_{2}$ and used to propagate virus stocks and to measure virus titers by plaque assays. Ribavirin passage experiments: monolayer cells were infected with virus stock in $2 \%$ FBS DMEM at a multiplicity of infection (MOI) of 100 PFU per cell, then absorbed for $1 \mathrm{~h}$ at $37^{\circ} \mathrm{C}$, and washed extensively to remove unabsorbed virions. Media with or without ribavirin of $400 \mu \mathrm{M}$ was added. After $48 \mathrm{~h}$, cells were lysed by freeze-thawing three times and cell debris was eliminated by centrifugation. The culture supernatant purified was used to infect fresh BHK-21 cells according to the same protocol. This procedure was repeated for five cycles; at time of harvest, aliquots collected from both cells and supernatants were frozen at each passage and designated from $\mathrm{Rp}_{1}$ to $\mathrm{Rp}_{5}$, respectively. Since untreated control virus population, designated from $\mathrm{P}_{1}$ to $\mathrm{P}_{5}$, was fairly constant in the parallel passage experiments, therefore, only $\mathrm{P}_{5}$ was utilized to be a control. Each of $\mathrm{Rp}_{1}$ to $\mathrm{Rp}_{5}$ presents different virus population.

Plaque assays. Virus was titered by standard plaque assay (Bachrach et al., 1957), with some modification. Briefly, Confluent monolayer of BHK-21 cells in 12-well plates as well as serial 10-fold dilutions FMDV virus in $2 \%$ FBS medium were prepared, $0.1 \mathrm{ml}$ of virus suspension was added to each well in triplicate for each dilution after removal of the medium. Infected cultures were then incubated at $37^{\circ} \mathrm{C}$ for $90 \mathrm{~min}$ with gently shaking every $15 \mathrm{~min}$ to assure uniform adsorption. Following this, cell monolayers were overlaid with $2 \mathrm{ml}$ maintenance medium containing $1.5 \% \mathrm{CMC}$ and incubated at $37^{\circ} \mathrm{C}$ in a $5 \% \mathrm{CO}_{2}$ incubator until plaque formed. Plaques were stained using $0.2 \mathrm{ml} 2 \%$ crystal violet in $10 \%$ formaldehyde and recorded after washing the monolayer with tap water.

Western-blotting analysis. Proteins extracted from the BHK-21 cells of T-25 flasks infected with untreated control virus $\mathrm{p}_{5}$ and $\mathrm{Rp}_{1}$ to $\mathrm{Rp}_{5}$ were directly subjected to $12 \%$ sodium dodecyl sulphate (SDS)-polyacrylamide gel, electrotransferred onto a nitrocellulose membrane, blocked with 5\% nonfat milk in PBS, and reacted with rabbit anti-FMDV serum as primary antibodies. Alkaline phosphatase (ALP)-conjugated goat anti-rabbit immunoglobulin $\mathrm{G}$ antibody (sigma) was used as the secondary antibody. After washing three times with TBST, containing $0.15 \%$ Tween-20, membrane-bound antibodies were detected with Nitro Blue tetrazolium/5-bromo-4chloroindol-2-yl phosphate.

Real-time RT-PCR Assay. The viral RNA was quantified using real time reverse transcription polymerase chain reaction (RT-PCR). TaqMan one-step quantitative RT- PCR assay was performed using a pair of primers FMDV-1 5'-GAACACATTCTTTACACCAGG AT-3' and FMDV-2 5'-CATATCTTTGCCAATCAACATCAG-3', which amplify a 121 bp DNA fragment, targeting the viral 3D gene encoding RNA-dependent RNA polymerase (nt 7184-7304 of Akesu/58 strain, GenBank accession number AF511039) and a TaqMan probe 5'-FAM-ACAACCTACCGCCGAGCCAATTC-TA MRA-3', corresponding to the region from nucleotides 7267-7289. The RT-PCR was performed with the Platinum ${ }^{\circledR}$ Quantitative RTPCR ThermoScript ${ }^{\mathrm{TM}}$ one-step Mastermix Reagents Kit (invitrogen) following the manufacture's protocol. Reverse transcription and amplification were carried out using Rotor-Gene 2000 Real-Time thermal cycler (Corbett). To compensate for variations inherent in sample preparation and reverse transcription the in vitro synthesized RNA templates were used as a dual control along with the copy number of viral RNA molecules, which is more reliable than reported RT-PCR employing a DNA-based standard. Optimal parameters were: cDNA synthesis: $5030 \mathrm{~min}$, the thermal profile for PCR was $95^{\circ} \mathrm{C}$ for $5 \mathrm{~min}$, followed by 40 cycles of $94^{\circ} \mathrm{C}$ for $30 \mathrm{~s}$ and $60^{\circ} \mathrm{C}$ for $90 \mathrm{~s}$. Each sample had three replicates and all reactions repeated three times independently to ensure the reproducibility of the results.

RT-PCR, molecular cloning, and sequencing. Total RNA was extracted from the BHK-21 cells infected by untreated control virus $\mathrm{p}_{5}$ and $\mathrm{Rp}_{1}$ to $\mathrm{Rp}_{5}$ using RNeasy Mini Kit (Qiagen, Hilden, Germany) according to the manufacture's instructions. The first strand cDNA was synthesized using an optimized protocol and a SuperScript ${ }^{\mathrm{TM}}$ Reverse Transcriptase (invitrogen) and PCR was performed with pfuultra ${ }^{\mathrm{TM}}$ High-Fidelity DNA polymerase (stratagene). Primers for amplification of the 3D polymerase-encoding region were 5'-CA CCATGGGGTTGATTGTTGACA-3' (positions 6639-6661 of FMDV genome) and 5'-TGCGTCACCGCACACGGCCGT-3' (positions 80488028). Primers for amplification of the nucleocapsid protein P1encoding region were 5'-CACCATGGGAGCCGGGCAAT-3' (positions 
1656-1675 of FMDV genome) and 5'-CAAAAGCTGTTTTGCG GGTGCC-3' (positions 3863-3842). The PCR products were ligated into a pGEM-T plasmid vector (promega, Madison, Wis.) and transformed into Escherichia coli DH5 bacteria and plasmid DNA was prepared from independent bacterial colonies. Plasmid DNA was sequenced in both orientations by (terminal deoxynucleotidyltransferase) Sanger di-deoxy chain termination method using the commercial (universal) primers SP6 and T7(promega). RT-PCRs were tested without RNA as a control to eliminate falsepositive results.

\section{Results}

Cell morphology observation of antiviral effect of ribavirin. Equal volume supernatant harvested from untreated control infected-cells and ribavirin passage experiments were inoculated onto a BHK-21 cell monolayer, and virus multiplication and cytopathic effect was used to evaluate the antiviral efficiency of ribavirin. Cytopathic effects (CPE) were observed in untreated control $\mathrm{P}_{5}$ virus-infected $\mathrm{BHK}-21$ cells beginning at 6hpi, and the cell sheet was complete destroyed at $8 \mathrm{hpi}$. In contrast, CPE were first observed in from $\mathrm{Rp}_{1}$ to $\mathrm{Rp}_{3}$-infected BHK-21 cells at 17, 23, 30 hpi, respectively. Furthermore, $\mathrm{Rp}_{4}$ and $\mathrm{Rp}_{5}$ infection did not induce cytopathic effect at all until $48 \mathrm{~h}$ postinfection. These results indicated that ribavirin treatment for FMDV caused dramatically inhibition of multiplication in cell cultures (Fig. 1).

Effect of ribavirin on infectious FMDV production. Indicated supernatants were inoculated onto a BHK-21 cell monolayer in 12-well plates for $1.5 \mathrm{~h}$ and then overlaid, and a plaque assay was performed to quantitate the yield of infectious virions in pooled supernatants collected during the ribavirin passage experiments. A large number of plaques were observed using untreated control virus $\mathrm{p}_{5}$, in contrast, the significant decrease by 2- and 2.5-fold of plaque numbers was acquired along with supernatants of $\mathrm{Rp}_{1}$ to $\mathrm{Rp}_{3}$. No infectious virus was detected after the third passage by plaque assay on BHK-21 cells and after the fourth passage and subsequent passages in the absence of ribavirin did not allow recovery of infectious virus in the supernatant, demonstrating viral infectivity was abolished completely and virus populations were forced into extinction.

\section{FMDV protein expression inhibition in infected cells with} supernatants of ribavirin passage experiments. The results of western blot assay showed that levels of FMDV translation were sharply reduced in BHK-21 cells infected with $\mathrm{Rp}_{1}$ to $\mathrm{Rp}_{3}$, and complete abrogation of protein expression was observed after the fourth passage of ribavirin passage experiments, which was consistent with the result of plaque assay, indicating the levels at viral proteins synthesis of virus populations were eliminated in infected-cells, or, the transcripts produced from viral genome treated with ribavirin were not functional.

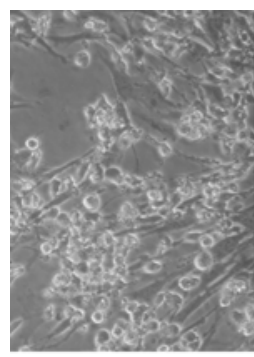

(A)

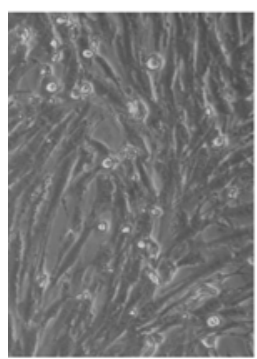

(D)

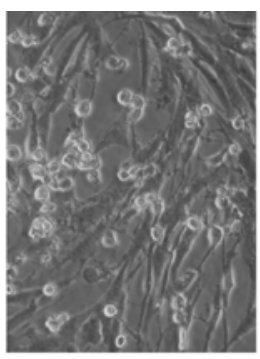

(B)

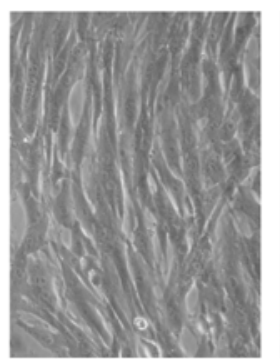

(E)

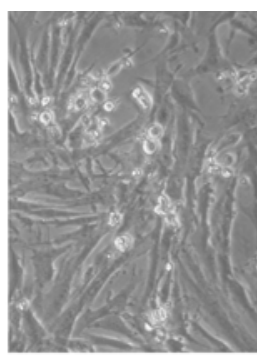

(C)

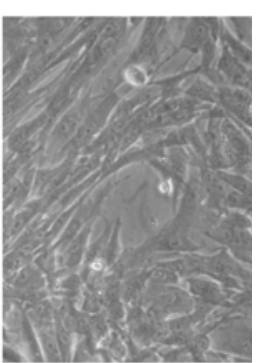

(F)
Fig. 1. Morphological features of the BHK-21 cells infected by untreated control virus $\mathrm{p}_{5}$ and $R \mathrm{p}_{1}$ to $\mathrm{Rp}_{5}$. $(\times 200)$. (A) untreated control virus $\mathrm{p}_{5}$ infection (7 h p.i.), (B) $\mathrm{Rp}_{1}$ infection (19h p.i.), (C) $\mathrm{Rp}_{2}$ infection (24h p.i.), (D) $\mathrm{Rp}_{3}$ infection (30 h p.i.), (E) $\mathrm{Rp}_{4}$ infection (48 h p.i.), (F) $\mathrm{Rp}_{5}$ infection (48 h p.i.).

Ribavirin treatment inhibits the levels of total viral RNA. Total RNA was extracted from the BHK-21 cells infected with untreated control virus $\mathrm{p}_{5}$ and $\mathrm{Rp}_{1}$ to $\mathrm{Rp}_{5}$ and viral RNA level was determined by real-time RT-PCR. Compared to untreated control infected-cells (defined as 100\%), levels of FMDV RNA replication, including plus-and minus-stranded, was sharply reduced to as little as $56,20,18,0.02,0 \%$ and 83 , $88,9,2,0 \%$, respectively, versus the passage number from $\mathrm{Rp}_{1}$ to $\mathrm{Rp}_{5}$. After the fourth passage subsequent passages in the absence of ribavirin did not allow detection of intracellular viral RNA. It is clear that the accumulation of plus and minus strands was ceased, which means that ribavirin treatment in consecutive passage experiments substantially reduce and even abrogate replication activity of viral population.

Mutation frequency of FMDV suggests error catastrophe. To evaluate the spectrum of mutations induced by ribavirin, we analyzed sequences obtained from independently cloned cDNAs of FMDV P1- and 3D-encoding region, from virus growth in the presence or absence of $400 \mu \mathrm{M}, 1,000 \mu \mathrm{M}$ ribavirin. Each value represents the 10 clones sequenced from each population. Meanwhile, virus infectivity loss as well as loss of total viral RNA were examined by plaque assay and real-time RT-PCR assay, respectively. A total of 108,540 nucleotides (nt) were sequenced. to obtain a representative population of mutations. The data were shown in (Table 1). The mutation cover more transitions of $\mathrm{C}$ to $\mathrm{T}$ and $\mathrm{G}$ to $\mathrm{A}$ and very fewer transversions, such as $\mathrm{C}$ to $\mathrm{G}, \mathrm{G}$ to $\mathrm{C}$ and $\mathrm{C}$ to $\mathrm{A}$, 
Table 1. The antiviral effects of ribavirin can be directly attributed to error catastrophe/lethal mutagenesis

\begin{tabular}{lccc}
\hline & Normal & $400 \mu \mathrm{M}$ & $1,000 \mu \mathrm{M}$ \\
\hline virus infectivity loss & - & $79 \%^{*}$ & $99.7 \%^{*}$ \\
Loss of total viral RNA & - & $44 \%^{*}$ & $8 \%^{*}$ \\
Mutation rate of 3D in ribavirin-treated RNA virus population & 0.7 & $2.4^{*}$ & $5.6^{* *}$ \\
Mutation rate of P1 of ribavirin- treated RNA virus population & 2.1 & $8.9^{*}$ & $22.5^{* *}$ \\
\hline
\end{tabular}

Note: Mutations per 10,000 nt sequenced.The total mutations found in the P1- and 3D-encoding region of FMDV derived from the infected-cells in the presence or absence $400 \mu \mathrm{M}, 1,000 \mu \mathrm{M}$ ribavirin include more transitions of $\mathrm{C}$ to $\mathrm{T}$ and $\mathrm{G}$ to $\mathrm{A}$ and very fewer transversions, such as $\mathrm{C}$ to $\mathrm{G}, \mathrm{G}$ to $\mathrm{C}$ and $\mathrm{C}$ to $\mathrm{A}$. The virus infectivity and total viral RNA of untreated control virus population was set to $100 \%$. Data were the average of three individual experiments. ${ }^{*} p<0.01,{ }^{*} p<0$. 001, compared to untreated control virus $\mathrm{P}_{5}$ infection (two-tailed, unpaired Student's t-test).

Table 2. The amino acid mutations distribution caused by ribavirin treatment in structural protein P1- and RNA-dependent RNA polymerase $3 \mathrm{D}$-encoding region

\begin{tabular}{llcl}
\hline & & Mutation types and positions & \\
\hline RNA-dependent & Pro $\rightarrow$ Ser (22,112) & Ser $\rightarrow$ Pro (140) & Ile $\rightarrow$ Met (193) \\
RNA polymerase & Ala $\rightarrow$ Val (242) & Ala $\rightarrow$ Thr (251) & Asn $\rightarrow$ Asp (261) \\
3D region & Asn $\rightarrow$ Lys (463) & & Gly $\rightarrow$ Arg (197) \\
\hline Structural protein & Ala $\rightarrow$ Gly (142) & Thr $\rightarrow$ Ile (179) & Asp $\rightarrow$ Asn (660) \\
P1 region & Ile $\rightarrow$ Thr (480) & Ala $\rightarrow$ Val (638) Ala (674) \\
\hline
\end{tabular}

Alignment of the predicted $3 \mathrm{D}^{\mathrm{pol}}$ and structural protein sequences of 20 clones was performed using an appropriate program. A total of seven type of amino acid mutations were made in 3D. Ten type of amino acid mutations were presented in $\mathrm{P} 1$, and $70 \%$ of that was located in VP1 region.

Ribavirin incorporation increased the mutation rate up to 8and 11-fold $(p<0.001)$ in 3D and P1 at a single round of virus infection in the presence of $1,000 \mu \mathrm{M}$ ribavirin, respectively, and caused a $99.7 \%(p<0.01)$ loss in viral infectivity versus the control experiment without ribavirin. Obviously, a direct correlation existed between the increase in mutation rate caused by ribavirin and the antiviral activity of the compound. Furthermore, protein sequence analysis proved that many amine acid mutations were found within 3D and $\mathrm{P} 1$ region. The data were shown in (Table 2). Due to the highly structured and multifunctional of RNA polymerase, polymerase sequence is general highly conserved, and a few mutations, especial in active site, probably result in the activity decline or complete loss. At the same time, capsid mutation likely lead to unencapsidation of the genomic RNA or non-viable virus progeny.

\section{Discussion}

RNA viruses were considered on the edge of error catastrophem (Stuart, 1996), because of the high replication error-rate and lack of proof-reading mechanism of RNA polymerase, including human immunodeficiency virus (HIV), human influenza virus A, vesicular stomatitis virus (VSV), foot-and-mouth disease virus (FMDV) and poliovirus. The error catastrophe theory has been proposed that once the error threshold, a critical error rate, forecasted from theoretical studies, is reached, even a slight increase in mutation frequency should cause a dramatic reduction in virus viability (Jason and Craig, 2002). Ribavirin triphosphate, a analog of GTP, can be incorporated into poliovirus genome and match with cytidine as well as uridine with equal efficiency in the RNA replication, promoting transitions of $\mathrm{G}$ to $\mathrm{A}$ as well as $\mathrm{C}$ to $\mathrm{U}$, reduces infectious poliovirus production to as little as $0.00001 \%$ in cell culture (Shane et al., 2000). In contrast, the well documented antiviral effect of ribavirin against FMDV was not directly associated with dramatic increases in virus mutation frequency.

In this paper, ribavirin was regarded as the antiviral reagent and FMDV as a model RNA virus by ribavirin passage experiments. It has been demonstrated that ribavirin significantly inhibits the production of infectious FMDV. However, its mechanism of action is not fully understood. Furthermore, in the ribavirin passage experiments infectious virus was not detected after the third passage by plaque assay and after the fourth passage intracellular FMDV RNA was undetectable by real-time RT-PCR. Meanwhile, no detectable viral proteins were observed after the fourth passage. At the fourth passage of consecutive passages in the absence of ribavirin did not allow recovery of infectious virus in the supernatant or detection of intracellular viral RNA. Furthermore, since FMDV presumably replicates via a minus-stranded RNA intermediate, 


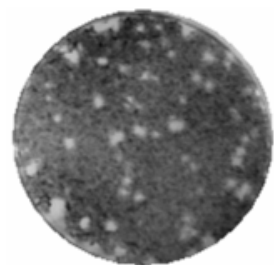

(A)

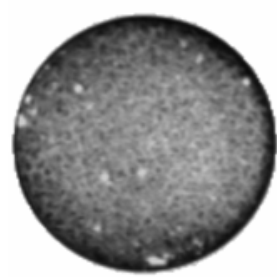

(D)

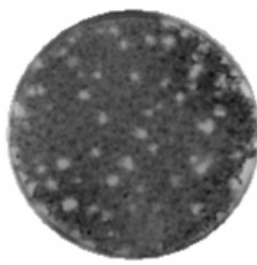

(B)

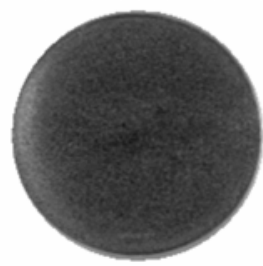

(E)

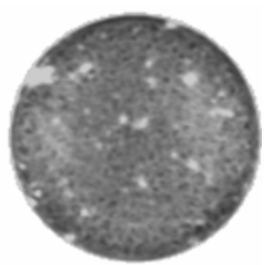

(C)

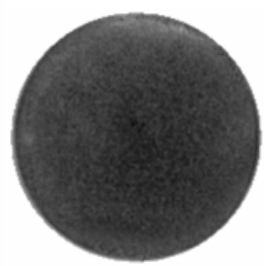

(F)
Fig. 2. Infectivity assays of indicated supernatants was performed by plaque assay. (A) 10-fold dilution of untreated control virus titer, (B) $\mathrm{Rp}_{1}$ titer, (C) $\mathrm{Rp}_{2}$ titer, (D) $\mathrm{Rp}_{3}$ titer, (E) $\mathrm{Rp}_{4}$ titer, (F) $\mathrm{Rp}_{5}$ titer. Supernatants of both the untreated control and $\mathrm{Rp}_{1}$ to $\mathrm{Rp}_{5}$ were titrated for infectious FMDV virions by plaque assay on BHK-21 cells as detailed in materials and methods.

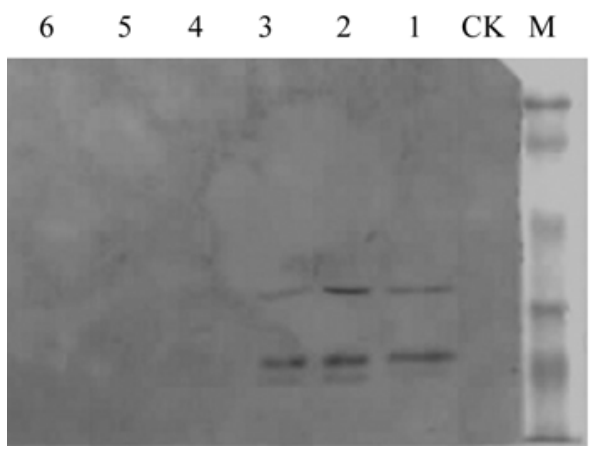

MWkDa
118.0
85.0
47.0
36.0
26.0
20.0

Fig. 3. Western blot analyses of FMDV antigen expression in BHK-21 cells infected by untreated control virus $\mathrm{p}_{5}$ and $\mathrm{Rp}_{1}$ to $\mathrm{Rp}_{5}$. Lane $\mathrm{CK}, \mathrm{BHK}-21$ cells; Lane 1: untreated control virus $\mathrm{p}_{5}$ infection; Lane 2: $R p_{1}$ virus infection; Lane 3: $R p_{2}$ virus infection; Lane 4: $\mathrm{Rp}_{3}$ virus infection; Lane 5: $\mathrm{Rp}_{4}$ virus infection; Lane 6: $\mathrm{Rp}_{5}$ virus infection; Lane $\mathrm{M}$, Protein Marker.

which is synthesized from plus-stranded genomic RNA, specific detection of minus-stranded FMDV RNA is very important to prove active replication in the infected cells. In $\mathrm{Rp}_{5}$-infected cells the synthesis of minus-stranded was ceased completely. All of this suggested that FMDV was forced into extinction. Whether the antiviral effect of ribavirin was directly correlated with its mutagenesis activity (lethal mutagenesis)? With this aim, we determined the mutation frequency in ribavirin-treated viral population and compared these values with those of an untreated control virus population. So the mutation frequency that leads to error catastrophe was measured by sequencing the P1- and 3D-encoding region of FMDV after a single round of virus infection in the presence
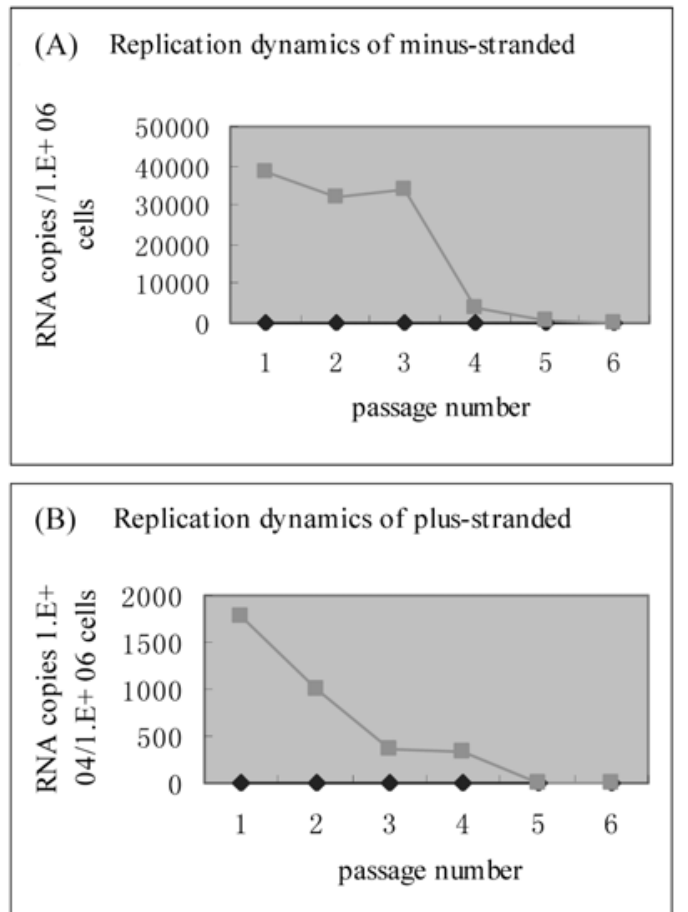

Fig. 4. The levels of viral RNA replication dynamics of minusstranded (A) and plus-stranded (B) was determined in infectedcells by untreated control virus $p_{5}$ and $R p_{1}$ to $R p_{5}$ with a rapidly and accurately real-time RT-PCR assay. 1. untreated control virus $\mathrm{P}_{5}$ infection; $2^{*} . \mathrm{Rp}_{1}$ virus infection, $3^{* *} . \mathrm{Rp}_{2}$ virus infection, $4^{* *}$. $\mathrm{Rp}_{3}$ virus infection; $5^{* *}$. $\mathrm{Rp}_{4}$ virus infection; $6^{* *} . \mathrm{Rp}_{5}$ virus infection. Each value represents the average of three independent experiments. ${ }^{*} p<0.01,{ }^{* *} p<0.001$ compared to untreated control virus $\mathrm{P}_{5}$ infection (two-tailed, unpaired Student's t-test).

or absence $400 \mu \mathrm{M}, 1,000 \mu \mathrm{M}$ ribavirin because several mutations were lethal for viral genome and not be transmitted to the next passage. For each sample and gene region we sequenced a similar number of clones derived from three independent RT-PCR reactions. At the concentration of $1,000 \mu \mathrm{M}$, ribavirin had a more strong antiviral effect. The sequencing data showed that a high mutation rate, up to 5.6/ $10,000 \mathrm{nt}$ and 22.5/10,000 nt in 3D and $\mathrm{P} 1$ region $(p<0.001)$ respectively, compared to $0.7 / 10,000 \mathrm{nt}$ and $2.1 / 10,000 \mathrm{nt}$ of wt virus, was found in viral RNA synthesized in a single round of virus infection in the presence of ribavirin and caused a $99.7 \%(p<0.01)$ loss in viral infectivity of virus population compared to parallel untreated control population, which demonstrating that a direct correlation existed between the mutagenic activity of ribavirin and the antiviral activity of the compound. Besides, a large number of amino acid mutations predicted from nucleotide sequence mutations were proved, and some of mutated 3D polymerase might be completely non-functional, and some of mutated P1 structural protein did not encapsidate the genome RNA, which is responsible for lethal mutagenesis.

The antiviral mechanism of ribavirin covers some viral 
targets, such as RNA polymerization and RNA capping or induce lethal mutagenesis of viral genomes (Delphine Benarroch et al., 2004) and correlates with IMPDH inhibition. However, it has been suggested that inhibition of IMPDH may not be enough for antiviral activity (Shane et al., 2000). In addition, FMDV mRNA is cap-independent and not influenced by the 2-O-methyltransferase and capping enzyme. To FMDV, virus RNA polymerase was selectively inhibited by the ribavirin triphosphate, which may be a potentially antiviral strategy. Future efforts will be directed to the development of in vitro assays for the enzymatic functions associated with RdRp to explore the effect of ribavirin. Multiple cycles of ribavirin incorporation could be allowed in single round infection in the presence of ribavirin, which does not terminate elongation of nascent RNA (Shane et al., 2000). In addition, ribavirin acts as a mutagenic ribonucleoside, resulting in a dramatic increase in the production of defective genome. At the same time, mutations of critical amino-acid residues in the polymerase actives sites could enhance the replication error-rate or disable the activity of polymerase (Stuart, 1996). We hypothesized that during FMDV replication the genomic and antigenomic templates, viral RNA and complementary RNA, sustain a lethal accumulation of errors. The following transcription and replication from these mutant templates results in increased genetic variation of genome RNAs that could affect their structure and function. Nevertheless, in light of our results for ribavirin the primary antiviral mechanism is attributable to lethal mutagenesis/error catastrophe.

In summary, the error catastrophe for FMDV offers a new paradigm for how to fight against viruses, namely, not merely by inhibiting their replication but rather by favoring it with an increased rate of mutation.

Acknowledgments We thank to D. Hui-yun CHANG for supplying FMDV.

\section{References}

Andrei, G. and De Clercq, E. (1993) Molecular approaches for the treatment of hemorrhagic fever virus infections. Antiviral Res. 22, 45-75.

Bachrach, H. L., Callis, J. J., Hess, W. R. and Patty, R. E. (1957) A plaque assay for foot-and-mouth disease virus and kinetics of virus reproduction. Virology 4, 224-236.

Cassidy, L. F. and Patterson, J. L. (1989) Mechanism of La Crosse virus inhibition by ribavirin. Antimicrob. Agents Chemother. 33, 2009-2011.

Cummings, K. J., Lee, S. M., West, E. S., Cid-Ruzafa, J., Fein, S. G., Aoki, Y., Sulrowski, M. S. and Goodman, S. N. (2001) Interferon and ribavirin vs interferon alone in the re-treatment of chronic hepatitis $\mathrm{C}$ previously nonresponsive to interferon: A meta-analysis of randomized trials. J. Am. Med. Assoc. 285, 193-199.

De Clercq, E. (2004) Antiviral drugs in current clinical use. $J$. Clin. Virol. 30, 115-133.
Delphine, B., Marie-Pierre, E., Laurence, M., Catherine, G., JeanLouis, R. and Bruno, C. (2004) A Structural Basis for the inhibition of the NS5 dengue virus mRNA 2-Omethyltransferase domain by ribavirin 5 -triphosphate. J. Biol. Chem. 279, 35638-35643.

Di Bisceglie, A, M., Thompson, J., Smith-Wilkaitis, N., Brunt, E. M. and Bacon, B. R. (2001) Combination of interferon and ribavirin in chronic hepatitis $\mathrm{C}$ : Re-treatment of nonresponders to interferon. Hepatology 33, 704-707.

Domingo, E., Martinez-Salas, E., Sobrino, F., de la Torre JC, Portela, A., Ortin, J., Lopez-Galindez, C., Perez-Brena, P., Villanueva, N., Najera, R., Vandepol, S., Steinhauer, D., Depolo, N. and Holland, J. (1985) The quasispecies (extremely heterogeneous) nature of viral RNA genome populations: Biological relevance-A review. Gene 40, 1-8.

Eriksson, B., Helgstrand, E., Johansson, N. G., Larsson, A., Misiorny, A., Noren, J. O., Philipson, L., Stenberg, K., Stening, G., Stridh, S. and Oberg, B. (1977) Inhibition of influenza virus ribonucleic acid polymerase by ribavirin triphosphate. Antimicrob. Agents Chemother. 11, 946-951.

Fernandez-Larsson, R., O'Connell, K., Koumans, E. and Patterson, J. L. (1989) Molecular analysis of the inhibitory effect of phosphorylated ribavirin on the vesicular stomatitis virus in vitro polymerase reaction. Antimicrob. Agents Chemother. 33, 1668-1673.

Isabelle Bougie and Martin Bisaillon (2004) The broad spectrum antiviral nucleotide ribavirin as a substrate for a viral RNA capping enzyme. J. Biol. Chem. 279, 22124-22130.

Jason, D. Graci and Craig E, Cameron. (2002) Quasispecies, error catastrophe, and the antiviral activity of ribavirin. Virology 298, 175-180.

Krilov, L. R. (2001) Respiratory syncytial virus: Update on infection, treatment, and prevention. Curr. Infect. Dis. Rep. 3, 242-246.

Maag, D., Castro, C., Hong, Z. and Cameron, C. E. (2001) Hepatitis C Virus RNA-dependent RNA Polymerase (NS5B) as a Mediator of the Antiviral Activity of Ribavirin. J. Biol. Chem. 276, 46094-46098.

Shane, C., David, M., Jamie, J. A., Weidong, Z., Johnson, Y. N., Zhi, H., Raul, A. and Craig, E. C. (2000) The broad-spectrum antiviral ribonucleotide ribavirin is an RNA virus mutagen. Nature Med. 6, 1375-1379.

Shane, C. and Raul, A. (2002) Implications of high RNA virus mutation rates: lethal mutagenesis and the antiviral drug ribavirin. Microbes Infection 4, 1301-1307.

Sidwell, R. W., Huffman, J. H., Khare, G. P., Allen, L. B., Witkowski, J. T. and Robins, R. K. (1972) Broad-spectrum antiviral activity of virazole:1-Beta-D-ribofuranosyl-1,2,4-triazole3-carboxamide. Science 177, 705-706.

Snell, N. J. (2001) Ribavirin-current status of a broad spectrum antiviral agent. Expert Opin. Pharmacother. 2, 1317-1324.

Streeter, D. G. (1973) Mechanism of action of 1-beta-Dribofuranosyl-1,2,4-triazole-3-carboxamide (Virazole), a new broad-spectrum antiviral agent. Proc. Natl. Acad. Sci. USA 70, 1174-1178.

Stuart, N. (1996) Life on the edge of catastrophe. Nature 384, 218-219.

Toltzis, P., O'Connell, K. and Patterson, J. L. (1988) Effect of phosphorylated ribavirin on vesicular stomatitis virus transcription. Antimicrob. Agents Chemother. 32, 492-497. 
Wray, S. K., Gilbert, B. E. and Knight, V. (1985) Effect of ribavirin triphosphate on primer generation and elongation during influenza virus transcription in vitro. Antiviral Res. 5, 39-48.

Wray, S. K., Gilbert, B. E., Noall, M. W. and Knight, V. (1985) Mode of action of ribavirin: effect of nucleotide pool alterations on influenza virus ribonucleoprotein synthesis.
Antiviral Res. 5, 29-37.

Wyde, P. R. (1998) Respiratory syncytial virus (RSV) disease and prospects for its control. Antiviral Res. 39, 63-79.

Xu, Z., Kuang, M., Okicki, I. R., Cramer, H. and Chaudhary, N. (2004) Potent inhibition of respiratory syncytial virus by combination treatment with $2-5 \mathrm{~A}$ antisense and ribavirin. Antiviral Res. 61, 195-206. 